\title{
Performance analysis of routing algorithms for optical burst switching
}

\author{
Óscar González de Dios ${ }^{1}$, Miroslaw Klinkowski², Carlos García Argos ${ }^{1}$, \\ Davide Careglio ${ }^{2}$, Josep Solé-Pareta ${ }^{2}$ \\ ${ }^{1}$ Telefonica I+D, c/Emilio Vargas, 6 \\ 28043 Madrid, Spain, \{ogondio, cgarcia\}@tid.es \\ ${ }^{2}$ Universitat Politècnica de Catalunya, Jordi Girona 1-3, Campus Nord, \\ 08034 Barcelona, Spain, \{mklinkw, careglio, pareta\}@ac.upc.edu
}

\begin{abstract}
In this paper we study the performance of several routing algorithms for optical burst switching network. The main aim is to find ways for performing dynamic load balancing and reduce congestion situation. In our analysis, we consider a common network scenario and, as a performance reference, we use the simple shortest path routing giving results of both analytical and simulation models. Therefore we propose many different routing algorithms based either on adaptive or non-adaptive strategies as well as distributed or isolated path selection. The obtained results highlight that none of the proposed strategies improve significantly the performance over the simple shortest path approach in the considered scenarios.
\end{abstract}

Keywords: optical burst switching, routing algorithms, performance evaluation.

\section{Introduction and motivation}

Optical burst switching (OBS) is a photonic network architecture directed towards efficient transport of IP traffic [1]. OBS pretends to be an intermediate solution for optical networks lying between Optical Circuit Switching (OCS) that nowadays in stage of standardization process and Optical Packet Switching considered as a farterm solution for optical networks. The potential advantages of OBS have caused a huge interest in the research in technologies to provide OBS functionality.

OBS architectures with limited (or even without) buffering capabilities are sensitive to traffic overloads. In particular, these traffic overloads cause burst drops, degrading network performance. Either a proper routing strategy with Traffic Engineering (TE) enhancement or adequate network dimensioning can help in the reduction of the congestion on specific links resulting in the increase of network throughput. Both approaches treat the congestion problem otherwise. Teng [2] proposes a TE approach to select the optimal paths for a given traffic matrix. Also, dimensioning the network considering a Shortest Path routing can also be performed [3]. The dimensioning approach fits the node and link capacities according to the matrix of actual traffic load demands and after such optimization it needs only either a 
simple Shortest Path algorithm or similar mechanism. However, some parts of such network may encounter congestion problem if the traffic demands change. Another approach for the congestion problem is to use routing strategies that improve network performance by means of online load balancing. Thodime [4] proposes an algorithm that statically computes link-disjoint alternate paths and dynamically selects one of the paths based on the collected congestion information. Thodime also proposes to periodically re-calculate the routes based on several metrics such as the physical distance, hops count, congestion information and link utilization. The authors in [5] present a proactive approach for tackling the problem of a burst contention based on adaptive use of multiple paths between edge nodes.

In this paper we investigate the routing strategies in OBS networks, starting from the Shortest Path approach to adaptive strategies that take into account network state. Moreover, we propose and study novel routing strategies with the aim of reducing burst loss probability. To asses the study, simulations are performed.

The rest of the paper is organized as follows. Section 2 describes the network scenario, the node characteristics and traffic modeling. In Section 3, we introduce a classification of the routing algorithms that are feasible for an OBS network. In Section 4 we consider the application of two static approaches and propose an analytical model to compute the burst loss probability for Shortest Path routing. Several adaptive routing approaches are considered in Section 5. Finally, Section 6 draws the conclusions of this work and outlines the further work.

\section{Network scenario}

In order to evaluate and compare the routing strategies, a reference scenario has been chosen. The topology is the 15 node NSFNet shown in Fig. 1. Every link has 16 data channels, with $10 \mathrm{Gbps}$ per channel. The nodes are enhanced with full wavelength conversion and 4 feed-forward FDLs. The average burst size is set to 40 kbytes, leading to $32 \mu \mathrm{s}$ of average burst length. The FDL granularity is the average burst length and up to $4 \mathrm{FDL}$ delays are allowed, leading to a maximum of $128 \mu$ s delay per burst. Nodes implement the Just Enough Time (JET) signaling protocol and a First InFirst Out resource reservation without preemption or priorities. The scheduling strategies that have been considered are Horizon/LAUC and Minimum starting void filling algorithms [6].

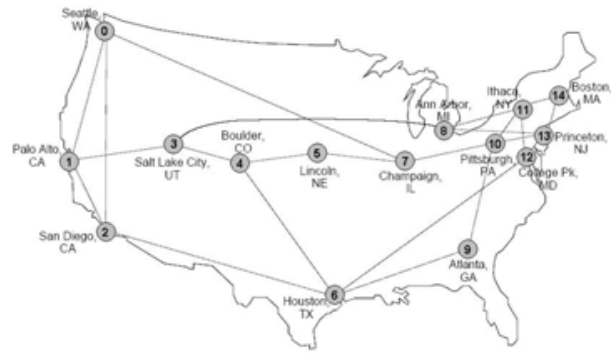

Fig. 1 15-node NSF network topology 
Regarding the traffic modeling, each node acts as an edge node, generating bursts that are sent with equal probability to all the other nodes. Both interarrival time and burst length are exponentially distributed. The study is performed varying the overall load introduced by the nodes, which is normalized with respect to the bandwidth of the links in order to allow a fair comparison of the results. The normalized load is defined as follows:

$$
\rho=\frac{T_{\text {node }}}{W \times B}
$$

Where $T_{\text {node }}$ is the mean traffic generated by each node, $W$ is the number of data wavelengths per link and $B$ the bandwidth of a single wavelength. Recall that the bandwidth of a link is $W \times B$.

Let $N$ be the number of nodes and $L$ the mean burst length (in bytes), the mean burst inter-arrival time (IAT) between each pair of nodes is

$$
I A T_{\text {node-node }}=\frac{L \times(N-1)}{W \times B \times \rho}
$$

And hence the overall network load is

$$
T_{\text {network }}=N \times W \times B \times \rho
$$

\section{Routing algorithms classification}

Two routing approaches exist: pure routing strategy like those used in IP networks and labeled routing strategy like MPLS. It is well-known that pure routing is not feasible for high speed networks requiring very fast lookup table processing. In OBS this problem is more evident for the huge amount of control packets to process. Therefore, setting up Labeled Path Switch in the so called Labeled OBS (LOBS) is considered the best approach; both Explicit Routing (ER) and Constraint-Based Routing (CBR) can be extended to provide and engineer the network resources [7]. In this paper we only consider ER solutions for the LOBS network. In particular, we consider that the LSPs between any pair of nodes are computed in advance, off-line, and downloaded to the nodes when the network is booted.

In such an environment, the ER can be set up in different way. Here we give a brief classification.

The path decisions can be grouped into two major classes: non-adaptive and adaptive. Non-adaptive ones do not base their routing decisions on measurements or estimates of the current traffic and topology, whereas adaptive ones do. This is sometimes called static routing and either single LSP or multi LSPs can be set up between any source node to any destination node. In case of multi LSPs, the source node usually balances the traffic among the LSPs. 
On the other hand, the adaptive approach needs to set up multi LSPs and attempts to change their path decisions to reflect changes in topology and the current traffic. Three different families of adaptive algorithms exist, namely centralized, isolated and distributed, which differ in the information they use. In the centralized solution, a single entity uses information collected from the entire network in an attempt to make optimal decisions. This solution is clearly unviable in wide area networks where the delays can be excessive. The other two solutions are more feasible. In the isolated approach, a local algorithm runs separately on each node, using local information, such as queue length. Finally, the third class of adaptive algorithms uses a mixture of global and local information.

Usually, the distributed approach is practical if decisions are taken only at the source node. In fact, it can receive congestion information from other nodes and take global optimal decisions selecting only the current best LSP or balance the use of multi LSPs according to a weight function. On the other hand, isolated approach is more beneficial if any node can decide the best route to the next node.

\section{Shortest Path and Equal Cost MultiPath analysis}

First, we consider two simple static routing techniques: the Shortest Path (SP) and Equal Cost Multi Path (ECMP). In the SP, only one LSP is available at each source node to get any destination node and we assume that the metric is the number of hops.

The SP strategy can be improved by using ECMP routing, which implements a multi LSPs strategy. For each possible source-destination pair, ECMP sets up all possible shortest LSPs (i.e., all those paths that have the same number of hops). Then, the source nodes balance the usage of the LSPs (i.e., each LSP is equally loaded).

\subsection{Iterative analytical model for Shortest Path routing}

We wish to analytically obtain the overall blocking probability for a given topology and traffic matrix, using Shortest Path routing. Some analytical methods have been already proposed in literature (e.g. [8]); here we are interested to propose a simple and iterative method. Its simplicity will give us, in future works, the possibility to easy enhance it in order to model adaptive routing strategies.

We assume that between every pair of nodes $i, j$ there is an offered traffic $T_{i, j}$. The path from node $i$ to node $j$ is fixed according to a Shortest Path routing strategy in the sense of number of hops. For the sake of simplicity, we will assume that all the links have the same number of wavelengths. Let us consider an OBS meshed network, where every node has several output links. We will consider JET scheduling and constant offset time. We will assume that burst arrivals follow a Poisson process. Thus, the link burst blocking probability $B$ is given by the Erlang-B formula

$$
B(W, \rho)=\left(\frac{\rho^{W}}{W !}\right) / \sum_{j=0}^{W} \frac{\rho^{j}}{j !}
$$


where $W$ is the number of wavelengths and $\rho$ is the traffic intensity in Erlangs in the link. The end-to-end blocking probability for a traffic flow $T_{i, j}$ is given by

$$
B_{i, j}=1-\prod_{k \in \text { path }(i, j)}\left(1-B_{k}\right)
$$

where $B_{k}$ is the blocking probability of the output link of a node in the path from node $i$ to node $j$ subject to a $\rho_{k}$ traffic intensity:

$$
B_{k}=B\left(W, \rho_{k}\right)
$$

We wish to obtain the overall blocking probability for a given network. The overall blocking probability is given by

$$
B_{\text {overall }}=\frac{\sum_{i=1}^{N} \sum_{j=1}^{N} B_{i, j} \cdot T_{i, j}}{\sum_{i=1}^{N} \sum_{j=1}^{N} T_{i, j}}
$$

The proposed iterative method to find the overall burst blocking probability $B_{\text {overall }}$ is shown below:

1. The first step is to apply the Dijkstra algorithm and calculate the Shortest Path, for every pair of nodes $(i, j)$, where $i=1, \ldots, N$, and $j=1, \ldots, N$, with $i \neq j$.

2. For each traffic intensity from node $i$ to node $j$, calculate the contribution of such traffic to link $k$

$$
T_{k(i, j)}^{n}=T_{i, j} \prod_{k \in \text { path }(i, j)}\left(1-B_{k}^{n}\right)
$$

At the first iteration ( $n=0), B_{k}$ is set to 0 for every link $k$. In the next steps, this value will be updated.

3. Once all the traffic intensities at every output link have been calculated, traffic intensities of the paths that cross link $k$ are summed

$$
\rho_{k}^{n}=\sum_{i=1}^{N} \sum_{j=1}^{N} T_{k(i, j)}^{n}
$$

4. For any $k$, calculate new values of $B_{k}{ }^{n}$ using equation (6) and determine the difference with the $B_{k}{ }^{n-1}$ calculated in the previous iteration

$$
D_{k}^{n}=\left|B_{k}^{n}-B_{k}^{n-1}\right|
$$

If $D_{k}{ }^{n}<D$ for all $k$, the iteration process has converged. If the iteration has not converged, update $n=n+1$ and go back to step 2 .

5. If the iteration has converged, the overall burst blocking probability is calculated using equation (7). 


\subsection{Simulation and analytical results}

The analytic results have been verified with the simulation. In order to provide more realistic results, the switching and processing times in the nodes are taken into account in the study. The BCP processing time in the intermediate nodes is set to 2.5 $\mu \mathrm{s}$. The speed of the switch matrix (switching time) is an important parameter in the scheduling process, and has been set to a conservative value of $1 \mu \mathrm{s}$. Fig. 2 depicts a comparison between the analytic results and the simulations, considering and not the realistic switching times. Results show that these switching times slightly increase the burst loss probability.

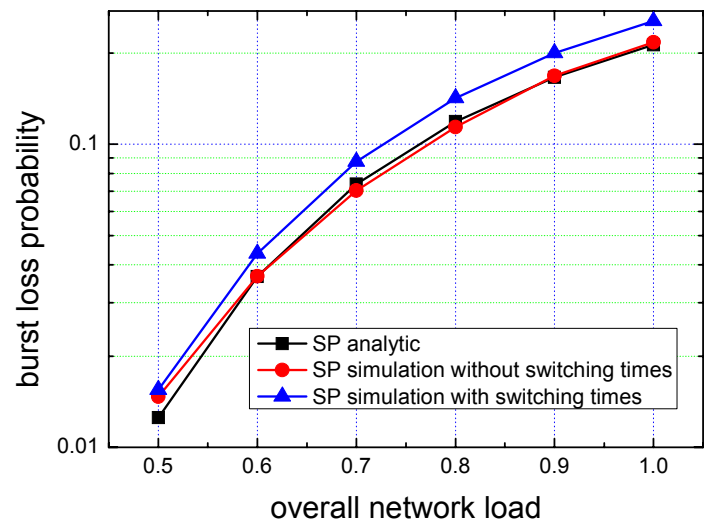

Fig. 2 Analytic vs simulation for SP routing without FDL buffering

Results for the SP and ECMP routing strategies are shown in Fig. 3. Four cases combining lack or presence of FDL buffering and Horizon or Minimum Starting Void scheduling are considered in obtaining these plots. It can be clearly seen that the use of buffers to avoid contention improves the overall blocking probability, even on high load conditions. The difference increases even more when Void Filling algorithms are used.
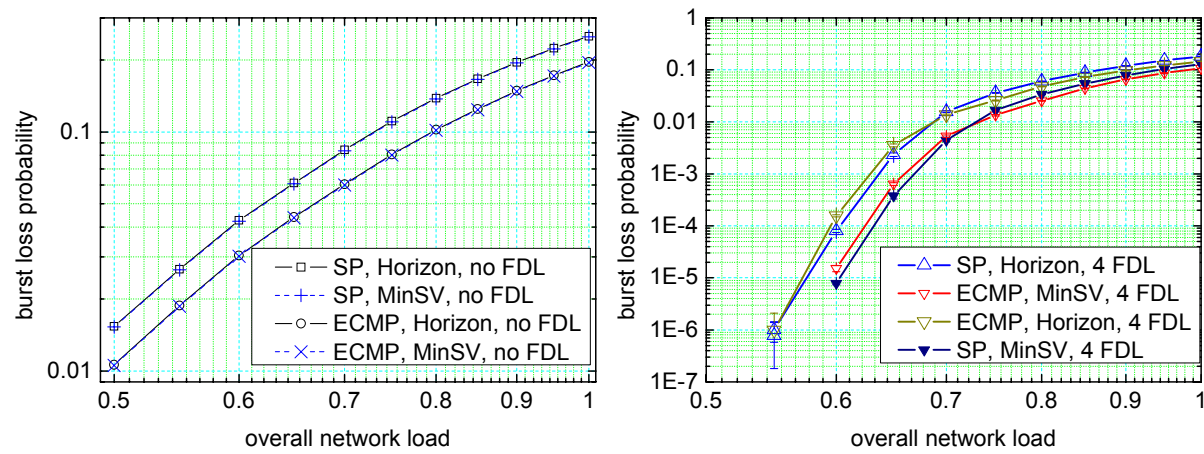

Fig. 3 Comparison between SP and ECMP routing strategies with and without FDL buffers 
The comparison between both routing strategies shows a very slight improvement on the burst loss probability parameter when no FDL are used and a performance loss when FDL buffers are used and the network load is low if ECMP routing is used to balance links load. The main reason of the slight difference is that, in this scenario, the network load is relatively better balanced when only one of the Shortest Paths among two nodes is considered then when using several shortest paths with no control over the quantity of traffic deflected. Although the global network load is more balanced with ECMP, alternate routes may get slightly overloaded and therefore increase their blocking probability, which causes a global increase in the network burst loss rate. However, the performance seems to be very dependent on the topology and some scenarios may benefit from the use of the ECMP routing.

\section{Adaptive routing}

For the following adaptive solutions, we consider that $k$ pre-established shortest LSPs between all source-destination pairs of nodes are available. These multi-LSPs are established only on knowledge of the network topology. Since it is possible to select one path from the set of $k$ available, each node (both source and intermediate) can make per-burst decision according to some parameters. Both isolated and distributed approaches are considered in the following.

\subsection{Isolated approach}

The isolated adaptive routing approach performs the path selection in consecutive nodes based on local node state information (like congestion conditions, actual link/buffer occupancy), i.e., each node can take a decision according to the state of its own output interfaces. It is a suboptimal solution since it only considers local information but provides good flexibility and no additional signaling is required. From this general concept, several specific algorithms can be inferred. Here we describe 4 different solutions: Path Excluding (PE), Bypass Path (BP), Multipath Routing with Dynamic Variance (MRDV), and Adaptive Multipath OBS Routing (AMOR).

5.1.1 PE description. The behavior of PE [9] algorithm is the following: each node always selects the less congested output queue among all the ports included in the set of available paths. This selection determines the next hop and excludes from the set of available paths all those paths that not include this hop in their route. Hence, from the $k$ original LSPs, each node is removing some paths as long as remains only one path.

5.1.2 BP description [9]. In this case, the source node selects one LSP from the $k$ available according to the state of its output queues and the minimum distance. The route can be modified only when traveling burst finds a congested link. In this case, the node tries to 'bypass' it using an intermediate node to reach the next hop.

5.1.3 MRDV description. MRDV [10] is a decentralized and dynamic link-state routing algorithm that balances the load in the network designed for IP Networks. The 
use of MRDV in OBS networks may help improve network performance. Thus, instead of pre-establishing only the shortest LSPs, longer paths are available to be used when the Shortest Paths are in high load condition. In [11] one of the authors investigated several metrics to balance the load among the possible paths, focusing on the stability of the load distribution.. MRDV assigns dynamic costs to all output links of a node in a traffic path and periodically searches for alternate output links in order to distribute network load among spare links.

5.1.4 AMOR description. This algorithm follows the idea that lies beneath the MRDV algorithm of assigning dynamic costs, but in a more proper way to adapt to OBS nodes particularities. These characteristics of OBS nodes limit their capability of reaction against congestion. While IP routers have finite memory that acts as a buffer, an OBS node may have FDL buffers which act as an optical memory but they are time limited. In the AMOR strategy, available paths are chosen more carefully than in MRDV strategy, as well as the load distribution function, which reacts to traffic profile changes.

5.1.5 Simulation results. From the Fig. 4 we can see that neither the PE nor BP isolated routing protocols help in resolving the congestion, as performance results of both strategies are only slightly better than the ones obtained for the SP algorithm. MRDV provided very similar results as ECMP due to the network topology and traffic profile, so readers are referred to Fig. 3, which show the ECMP behaviour. The AMOR strategy is still under study, and no definitive results are available yet.

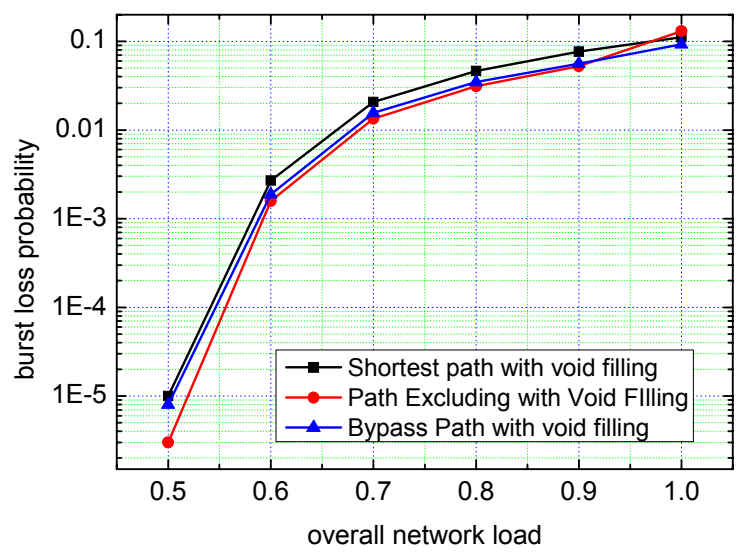

Fig. 4 Comparison among SP, PE and BP routing (nodes use 4 FDLs)

\subsection{Distributed approach}

The distributed adaptive routing approach, which performs the path selection in consecutive nodes based on global node state information. Each node collects the global state information that the other nodes send by means of flooding and applies a cost function to determine the lowest cost path. This solution introduces the problem 
of inaccuracy in network state information; in fact, routing decisions performed by such algorithm is optimal as long as this information perfectly represents the actual network state, what is impossible to achieve in real networks. Moreover, distributed routing involves additional signaling complexity so as to exchange the state information inside the network. Here we present one solution: the Distributed Path with $n$ alternatives (DP-n).

5.2.1 DP-n description. For this strategy, each node takes samples of its states (in our implementation the states are represented by the average utilizations of each output link) during a given period $T$. When this time expires, each node floods its collected data to all other nodes. At the same time, the node recalculates the cost of each multi LSPs using the last update messages received. The cost function $f$ at time $t$ for LSP $y$ between node $i$ and $j$ is

$$
f_{i, j}^{y}(t)=\alpha \sum_{x \in \text { path }(i, j)} \overline{U L}_{x}+(1-\alpha) f_{i, j}^{y}(t-T)
$$

where $U L_{x}$ is the average utilization of link $x$ during period $(t-T, t)$ and $\alpha$ is the memory factor, which takes into account previous periods.

Once the costs of every LSPs are computed, source nodes balance the traffic among a subset $n$ of the pre-established $k$ LSPs according to their costs.

5.2.2 Simulation results. In the following part we analyze the performances of the algorithm previously described. We have compared DP-1 and DP-4 with the burst loss probability obtained by using the SP algorithm. Four LSPs $(k=4)$ are preestablished for any source-destination pair.

Analyzing Fig. 5 it is really difficult to find a routing algorithm with better performance, as all the curves are really close. Moreover, the results show that DP-4 does not significantly improve the performances of the DP-1 algorithm.

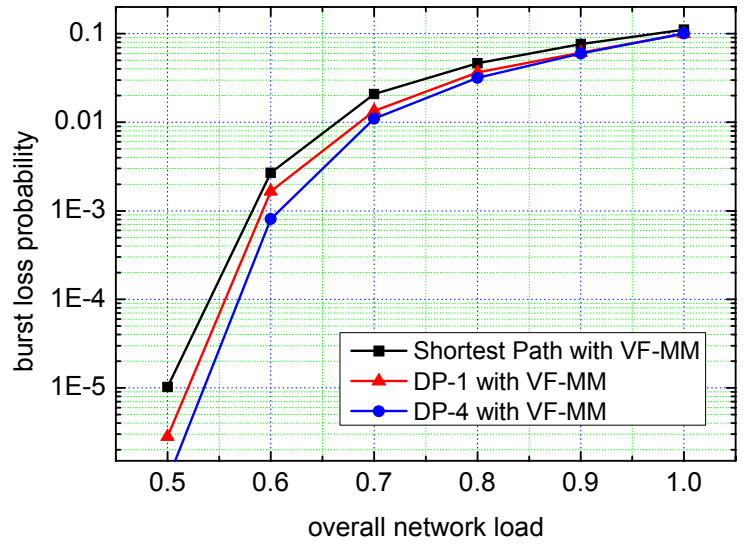

Fig. 5 Comparison among SP, DP-1 and DP-4 routing (nodes use 4 FDLs) 


\section{Conclusions and further work}

In this paper we have presented and compared several routing strategies aimed at improving OBS network performance. By means of simulation the algorithms under study were compared. Furthermore, a method to calculate the burst loss probability analytically with the shortest path strategy was presented and validated against the simulations. The main conclusion is that none of the proposed strategies improve significantly the performance over the simple shortest path approach for this network topology and traffic profile. Thus, it is questioned whether it is worth increasing complexity in the network routing in OBS. However, further scenarios that take into account a realistic network evolution must be studied to have more general results and conclusions. Specifically, one scenario with a dynamic traffic profile may benefit from the adaptive strategies presented in this work, and another scenario with link and node failures that unbalance the network and require a mechanism to automatically route the traffic in the most efficient manner.

Acknowledgments. This work has been partially funded by IST project NOBEL II (FP6-027305) and the MEC (Spanish Ministry of Education and Science) under the CATARO project (TEC2005-08051-C03-01).

\section{References}

1. C. Qiao, M. Yoo: Optical burst switching (OBS) - a new paradigm for an optical Internet. Journal of High-Speed Networks, vol. 8, no. 1, pp. 69-84, Mar. 1999

2. J. Teng, G. N. Rouskas: Routing Path Optimization in Optical Burst Switched Networks. IEEE 2005

3. C.M. Gauger,, M. Köhn, J. Zhang, B. Mukherjee: Network performance of optical burst/packet switching: the impact of dimensioning, routing and contention resolution. Beiträge zur 6. ITG Fachtagung Photonic Networks, Leipzig, 2005

4. G. P.V. Thodime, V. M. Vokkarane, and Jason P. Jue: Dynamic Congestion-Based Load Balanced Routing in Optical Burst-Switched Networks. IEEE Globecom 2003

5. S. Ganguly, S. Bhatnagar, R. Izmailov, C. Qiao: Multi-path adaptive optical burst forwarding. Proc. of the IEEE HPSR 2004, Phoenix, AR, Apr. 2004

6. J. P. Jue, V. M. Vokkarane: Optical Burst Switched Networks. Springer Science Optical Networks Series, 2005

7. C. Qiao. Labelel optical burst switching for IP-over-WDM integration. IEEE Com. Mag., vol. 38 , no. 9, Sep. 2000

8. Z. Rosberg, H.-L. Vu, M. Zukerman, J. White: Blocking Probabilities of Optical Burst Switching Networks Based on Reduced Load Fixed Point Approximations. Proc. of Infocom 2003, San Francisco, CA, Mar. 2003.

9. M. Klinkowski, F. Herrero, D. Careglio, J. Solé-Pareta: Adaptive routing algorithms for optical packet switching networks. ONDM 2005, Milan, Italy, Feb. 2005

10.F.J. Ramón Salguero, J. Andrés-Colas, J Enríquez-Gabeiras, G. García-de-Blas: Dynamic Routing Strategies to Postpone Network Congestion. Technical Report, COST 279, 2002

11. J. Aracil, O. González, J P. Fernández-Palacios, Routing strategies for OBS networks based on MRDV, ICTON 2005, Barcelona, Spain, July 2005 